\title{
Motor Unit Recruitment Strategies Are Altered during Deep-Tissue Pain
}

\author{
Kylie Tucker, ${ }^{1}$ Jane Butler, ${ }^{3}$ Thomas Graven-Nielsen, ${ }^{4}$ Stephan Riek, ${ }^{2}$ and Paul Hodges ${ }^{1}$ \\ Schools of ${ }^{1}$ Health and Rehabilitation Sciences and ${ }^{2}$ Human Movement Studies, The University of Queensland, St. Lucia, Queensland 4029, Australia, \\ ${ }^{3}$ Prince of Wales Medical Research Institute, Randwick, New South Wales 2031, Australia, and ${ }^{4}$ Center for Sensory-Motor Interaction, Aalborg University, \\ 9220 Aalborg, Denmark
}

Muscle pain is associated with decreased motor unit discharge rate during constant force contractions. As discharge rate is a determinant of force, other adaptations in strategy must explain force maintenance during pain. Our aim was to determine whether motor unit recruitment strategies are altered during pain to maintain force despite reduced discharge rate. Motor unit discharge behavior was recorded in two muscles, one with (quadriceps) and one without [flexor pollicis longus (FPL)] synergists. Motor units were recruited during matched low-force contractions with and without experimentally induced pain, and at higher force without pain. A total of 52 and 34 units were recorded in quadriceps and FPL, respectively, during low-force contractions with and without pain. Of these, 20 quadriceps and 9 FPL units were identified during both trials. The discharge rate of these units reduced during pain in both muscles [quadriceps: 8.7 (1.5) to $7.5(1.3) \mathrm{Hz}, p<0.001$; FPL: 11.9 (1.5) to 10.0 (1.7) Hz, $p<0.001$ ]. All remaining units discharged only with or without pain, but not in both conditions. Only one-third of the additional units recruited during pain (quadriceps $n=7 / 19$, FPL $n=3 / 15$ ) were those expected given orderly recruitment of the motor unit pool as determined during higher-force contractions. We conclude that reduced motor unit discharge rate with pain is accompanied by changes in the population of units used to maintain force. The recruitment of new units is partly inconsistent with generalized inhibition of the motoneuron pool predicted by the "pain adaptation" theory, and provides the basis for a new mechanism of motor adaptation with pain.

\section{Introduction}

Despite intensive investigation, only relatively simplistic theories have been proposed to explain changes in movement that occur with pain. The most widely accepted "pain adaptation" theory argues that movement amplitude and velocity of the painful segment are reduced by a combination of inhibition of agonist and facilitation of antagonist muscles (Lund et al., 1991). Although many observations during pain have been interpreted to support this theory (Graven-Nielsen et al., 1997; Svensson et al., 1997; Le Pera et al., 2001), others do not (Svensson et al., 1998; Sessle, 1999; Birch et al., 2000).

More recently the pain adaptation theory has been challenged by motor unit discharge behavior during experimental pain. Discharge rate of motor units is directly related to force production (Stuart and Enoka, 1983). However, discharge rate is decreased during contractions with matched force when pain is induced experimentally in the test muscle (Sohn et al., 2000; Farina et al., 2005). While decreased discharge rate has been considered to support the pain adaptation theory, it is not known how force is maintained under these conditions. The questions that must be addressed are, how can force be maintained when motor unit

Received 0ct. 28, 2008; revised April 6, 2009; accepted April 16, 2009

Financial support was provided by the National Health and Medical Research Council of Australia.

Correspondence should be addressed to Dr. Paul Hodges, National Health and Medical Research Council Centre of Clinical Research Excellence in Spinal Pain, Injury, and Health, School of Health and Rehabilitation Sciences, The University of Queensland, St. Lucia, QLD 4029, Australia. E-mail: p.hodges@uq.edu.au.

DOI:10.1523/JNEUROSCI.5211-08.2009

Copyright $\odot 2009$ Society for Neuroscience $\quad 0270-6474 / 09 / 2910820-07 \$ 15.00 / 0$ discharge rate is reduced, and what can this tell us about the physiological mechanisms that underpin changes in movement control with pain?

A number of possible mechanisms may assist force maintenance despite reduced discharge rate. It has been shown that adaptation of muscle fiber membrane properties is unlikely to account for force maintenance (Farina et al., 2008). Furthermore, synergist muscle activity that could compensate for reduced activity of painful muscle does not increase (Hodges et al., 2008). Other mechanisms may include simultaneous reduction in antagonist muscle activity, increased force contribution by movement of adjacent body segments, and/or altered recruitment of other motor units within agonist muscles.

The aim of these studies was to determine whether the motor unit recruitment strategy used to maintain force is altered with pain. In the first study (study A), pain was induced in the infrapatellar fat pad (Bennell et al., 2004) and motor unit activity recorded from the lateral and medial vasti muscles, which are synergists during knee extension. In the second study (study B), pain was induced in the flexor pollicis longus (FPL) and motor unit activity recorded from the same muscle. This muscle flexes the distal third of the thumb and was chosen as it has no synergists.

We hypothesized that during matched force tasks, in muscles both with and without synergists, (1) the discharge rate of motor units would decrease with pain; and (2) additional units would be recruited to maintain force when the discharge rates of original units are decreased. In addition, we hypothesized that (3) the 

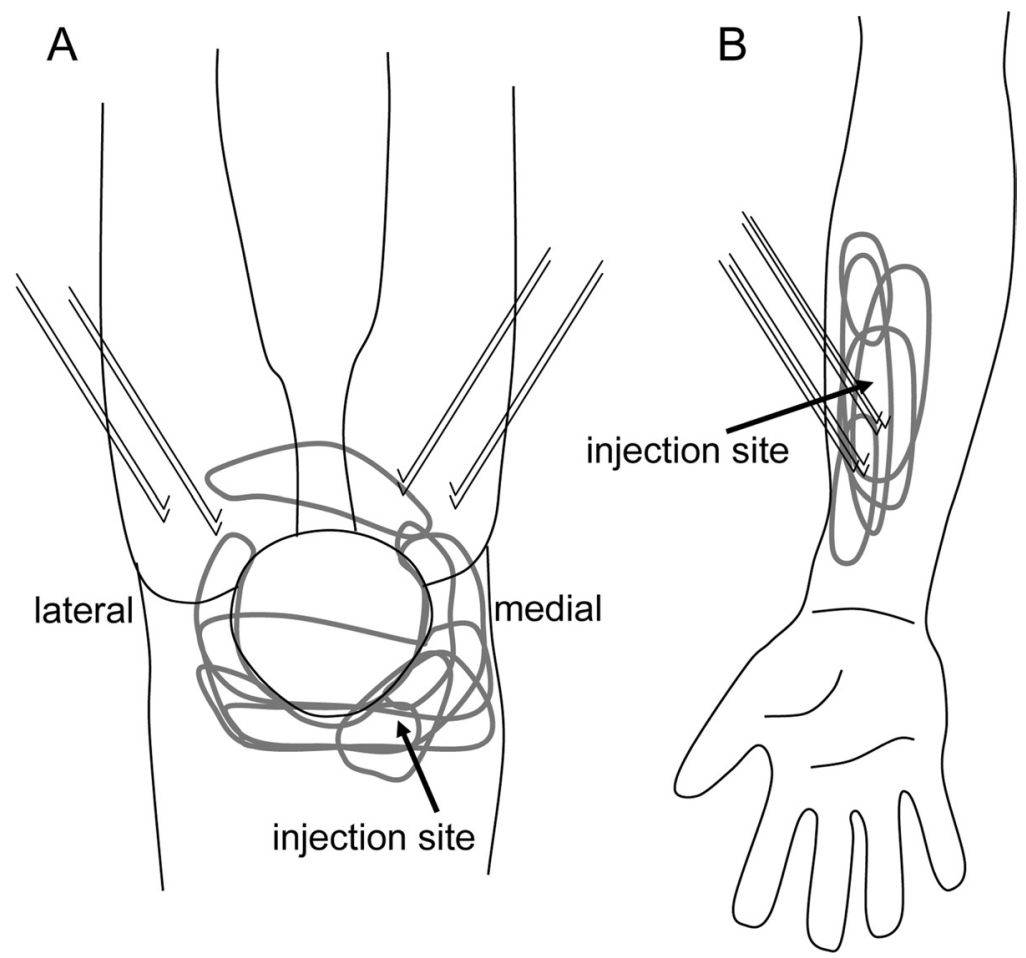

Figure 1. Area of pain and location of fine-wire electrodes. The injection site, approximate area of reported pain for each subject (gray), and location of fine-wire EMG electrodes are shown. Hypertonic saline $(5 \% \mathrm{NaCl})$ was injected into the patellafemoral fat pad in study $A$, and FPL in study B.

additional units would be the same as those recruited during a higher-force task in line with orderly recruitment of the motor unit pool and that (4) as a result of the decrease in discharge rate of some units and the recruitment of others, the net motor unit activity would not change during pain.

\section{Materials and Methods}

Fourteen volunteers participated in this series of experiments [age: mean (SD) study A: 29 (9) years, 4 males and 4 females; and study B: 30 (4) years, 6 males and 1 female]. One subject participated in both experiments. Subjects had no history of significant knee/leg pain for study A or arm/hand pain for study B. The Institutional Medical Research Ethics Committee approved the study, and all procedures conformed to the Declaration of Helsinki.

Electromyography. For study A, two pairs of intramuscular fine-wire electromyography (EMG) electrodes (two Teflon-coated $100 \mu \mathrm{m}$ stainless-steel wires with $0.5 \mathrm{~mm}$ insulation removed) were inserted into the medial (vastus medialis obliquus) and lateral (vastus lateralis) heads of the quadriceps. The needle was removed following insertion, leaving the wires in place (Fig. 1A). EMG data were preamplified 1000-10,000 times, bandpass filtered $(30 \mathrm{~Hz}$ to $2 \mathrm{kHz}$ ), notch filtered at $50 \mathrm{~Hz}$, and sampled at $5 \mathrm{kHz}$ using a Power1401 Data Acquisition System with Spike2 software (Cambridge Electronic Design).

For study B, two fine-wire electrodes (either three Teflon-coated 75 $\mu \mathrm{m}$ stainless-steel wires with $2 \mathrm{~mm}$ insulation removed $(n=4)$ or one Teflon-coated $100 \mu \mathrm{m}$ stainless-steel wire with $2 \mathrm{~mm}$ insulation removed and three $75 \mu \mathrm{m}$ Formvar-coated stainless-steel wires with cut ends) were inserted into FPL. The first electrode was inserted one-third of the distance between the radial styloid process and elbow crease, and the second $\sim 2 \mathrm{~cm}$ proximal to this point (Fig. $1 B$ ). This configuration allowed three to six possible pairs of intramuscular recording electrodes. An additional electrode (two Teflon-coated $75 \mu \mathrm{m}$ stainless-steel wires with $2 \mathrm{~mm}$ of insulation removed) was inserted into the belly of the antagonist muscle, extensor pollicis longus (EPL) in six of seven subjects. All electrodes were inserted with guidance of ultrasound imaging (12 $\mathrm{MHz}$, Logic 9, GE Healthcare). Separate pairs of wires were tested to identify those that provided the most selective single motor unit recordings for discrimination purposes. Data were amplified and filtered as for study A.

Experimental design. In both studies, subjects were positioned in a way to restrict the force produced from adjacent body segments. For study A, subjects sat fully supported with their leg relaxed over the end of a plinth. Upper thighs were strapped firmly to the plinth to avoid changes in hip position during knee extension. Isometric knee extension force was measured with a force transducer (Futek) that was secured to the plinth and attached via a strap just above the ankle. For study B, subjects sat with their forearm resting on the base of a hand support rig with their fingers and palm gripped around a vertical steel rod. The thumb was supported in the horizontal plane by an adjustable metal clamp positioned just proximal to the distal interphalangeal joint and a torque transducer aligned with the center of the joint. This set-up isolated torque production to flexion of the distal interphalangeal joint, which is produced solely by the FPL (Wood Jones, 1941).

For both studies, subjects were instructed to gently contract the test muscles until two to six single motor units fired consistently. Once this target level was established and recorded, subjects were asked to contract at a higher level to recruit two to three additional motor units. This contraction enabled us to identify the next units that would be expected based on orderly recruitment of the motoneuron pool. These target force (study A) or torque (study B) levels were presented on a feedback screen and matched by the subject before and during experimental pain. Subjects slowly increased force from rest to the target level, and then maintained this force for 10-20 s before slowly reducing force to rest. In all conditions the contractions were repeated three times. The order of force level (lower or higher) contractions was randomly allocated. Ten seconds of data from each of the contractions was used for further analysis. As a sensitive force transducer was used to measure force in study $\mathrm{A}$, maximum voluntary contractions (MVCs) were not measured; however, MVCs were performed in four of seven subjects in study B following complete resolution of pain.

Experimental pain. For study A pain was induced by single bolus injection of hypertonic saline $(0.25 \mathrm{ml}, 5 \% \mathrm{NaCl})$ into the infrapatellar fat pad (Bennell et al., 2004). For study B, a catheter $(0.8 \times 25 \mathrm{~mm}$; BD Venflon) was inserted (with ultrasound guidance) into FPL $\sim 3 \mathrm{~cm}$ proximal to the most proximal fine-wire electrode at the beginning of the experiment. A constant flow device (Asena Mark III, Alaris Medical Systems) was attached to the catheter just before the painful condition, and controlled the injection of hypertonic saline at $90 \mathrm{ml} / \mathrm{h}$ for $20 \mathrm{~s}$ followed by $6-9 \mathrm{ml} / \mathrm{h}$ for the remaining experimental pain period. Experimental muscle pain was recorded throughout the pain trial using a custom-made electronic $10 \mathrm{~cm}$ visual analog scale (VAS) whereby $0=$ "no-pain" and $10=$ "worst pain imaginable." In both studies, the test contractions commenced after the pain level reached $3 / 10$ on the VAS. The area of pain was also recorded by the subjects on a standardized figure (Fig. 1) following the pain trial.

Data analysis. Single motor units were identified based on morphology using Spike2 software (Cambridge Electronic Design) from the pairs of fine-wire electrodes that produced the recordings with the best discrimination between motor units throughout the trials. To ensure discrimination accuracy, motor unit interspike intervals were examined. Trials that contained abnormally short or long interspike intervals were reanalyzed on a spike-by-spike basis to check for discrimination accuracy. Averages of single motor unit and multiunit recordings were then triggered from the firing of each discriminated unit over the $10 \mathrm{~s}$ analysis 
period to generate a profile template of the motor unit morphology. These profiles were compared visually within subjects to determine whether the same unit was present in each contraction. Motor units that discharged for more than half of at least two of the three $10 \mathrm{~s}$ contractions within a condition were considered reliable for that condition. The mean discharge rate of all reliable motor units was recorded for comparison between conditions. Motor units from the two regions of the quadriceps muscle (vastus medialis obliquus and vastus lateralis) behaved similarly between conditions and were grouped for analysis purposes.

In study B, gross muscle activity (root mean square) was determined from the fine-wire electrode pair inserted into the EPL, and from the fine-wire electrode pair that recorded the greatest number of active motor units in the FPL.

Statistical analysis. Comparison of the discharge rate of motor units identified in both the no-pain and pain low-force trials, gross EMG and force level were made with paired $t$ tests (two tails). An independent $t$ test (two tails) was used to compare the discharge rate of the whole population of motor units recruited in the no-pain or pain low-force conditions. Data are presented as mean (SD) throughout the text and figures. Significance was set at $p<0.05$.

\section{Results}

The average knee extension force required to recruit two to six motor units was $10.3(7.5) \mathrm{N}$ (range 3.8-25.61 N). On average the force maintained during pain was 96.0 (4.0)\% of the no-pain level ( $p=0.3$ ). The average flexion torque at the distal interphalangeal joint of the thumb used to recruit two to six motor units (from four subjects) was 1.5 (0.7)\% MVC (range $0.8-2.4 \%$ MVC). On average the torque maintained during pain was 92.8 (8.4)\% of the no-pain level $(p=0.8)$.

The average pain rating reported during knee pain was 6.5 (1.7)/10, and during FPL pain was $3.8(0.8) / 10$. Pain was generally localized to the area surrounding the saline injection (Fig. 1). One subject reported mild discomfort at the site of one fine-wire insertion in the quadriceps throughout the study.

A total of 52 motor units were discriminated in the quadriceps at the lower force level, 33 of which were recruited in the contractions without pain and 39 with pain; $20(38 \%)$ were recruited in both conditions (Fig. 2A). In the FPL, 34 motor units were discriminated from the lower-force contractions, 19 of which were recruited during the contractions without pain and 24 with pain; $9(26 \%)$ of these units were recruited in both conditions (Fig. $2 B$ ).

The discharge rate of the units identified in both the low-force no-pain and pain conditions in the quadriceps and FPL decreased significantly with pain $(p<0.001)$. When the entire population of units recruited in either low-force condition was considered, the discharge rate also decreased significantly in the quadriceps $(p<0.001)$, and the FPL $(p=0.03)$. Representative and group data are shown in Figures 3-6. Interestingly, although the group data clearly show a decrease in discharge rate with pain in both muscles, of the 20 units identified during both the no-pain and pain lower-force contractions in the quadriceps, two motor units increased in discharge rate with pain [from $6.7(0.4)$ to $7.0(0.4) \mathrm{Hz}]$.

Several findings suggest that pain induced a change in motor unit recruitment. First, some motor units $(n=13 / 33$ in quadriceps and $n=10 / 19$ in the FPL) that were recruited during contractions without pain were not recruited during pain. Second, the derecruitment of this population of units coincided with the recruitment of a separate population of new units $(n=19 / 39$ in quadriceps and $n=15 / 23$ in FPL) during pain. Third, subjects also performed contractions at a higher force level during the no-pain condition to determine the next units expected based on orderly recruitment of the motoneuron pool. Unexpectedly, only

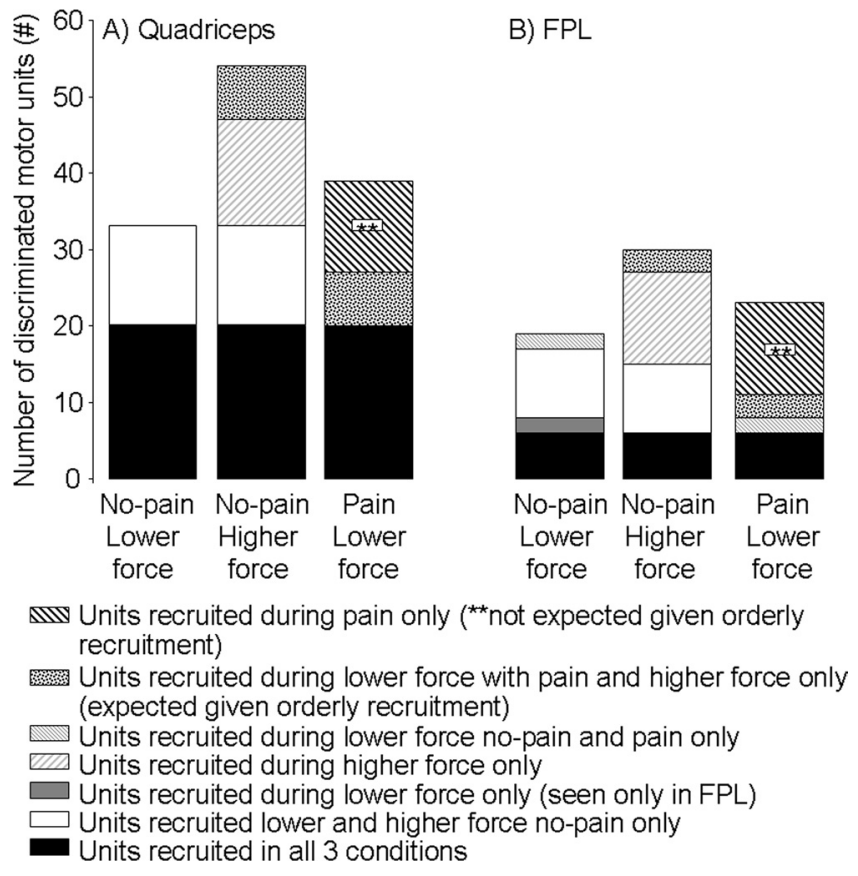

Figure 2. Number of discriminated motor units in quadriceps $(\boldsymbol{A})$ and $\mathrm{FPL}(\boldsymbol{B})$ during the three contraction conditions. A similar pattern of recruitment between conditions is seen in both muscles.

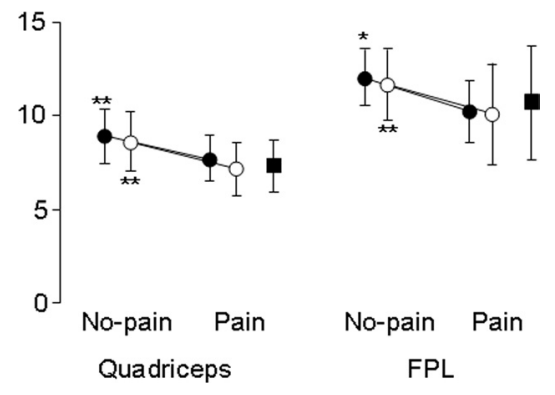

Figure 3. The discharge rate of motor units recruited in the quadriceps $(n=18)$ and FPL $(n=8)$ in trials both with and without pain decreased significantly with pain (closed circles). When the discharge rate of the whole population of units identified in either condition (open circles) was compared, a reduction in discharge rate was also identified in both muscles. The discharge rate of the units recruited during pain at the lower force level (closed squares) is also shown. ${ }^{*} p<0.05 ;{ }^{* *} p<0.001$. Mean and SD are shown.

7 of the additional 19 units in the quadriceps and 3 of the additional 15 units recruited during pain were the same as those additional units recruited at the higher force level (Fig. 2). Together these data demonstrate a change in recruitment that is inconsistent with uniform inhibition of the motoneuron pool.

These changes in motor unit recruitment represent group data from between two and four separate fine-wire recordings in each subject in the quadriceps ( $n=20$ fine-wire recording zones in total), and one fine-wire recording per subject ( $n=7$ finewire recording zones in total), in the FPL. Further detail of recruitment changes can be gained from analyzing changes in recruitment within a muscle compartment, and between compartments. In 5/20 of the fine-wire recording zones in the quadriceps and $3 / 7$ of the fine-wire recording zones in FPL, there was a change in recruitment where at least one unit was no longer recruited but substituted with the firing of at least one new unit during pain (e.g., Fig. 5, unit D not recruited and units B, F, and $G$ recruited during pain in FPL; and Fig. 6, unit A not recruited 


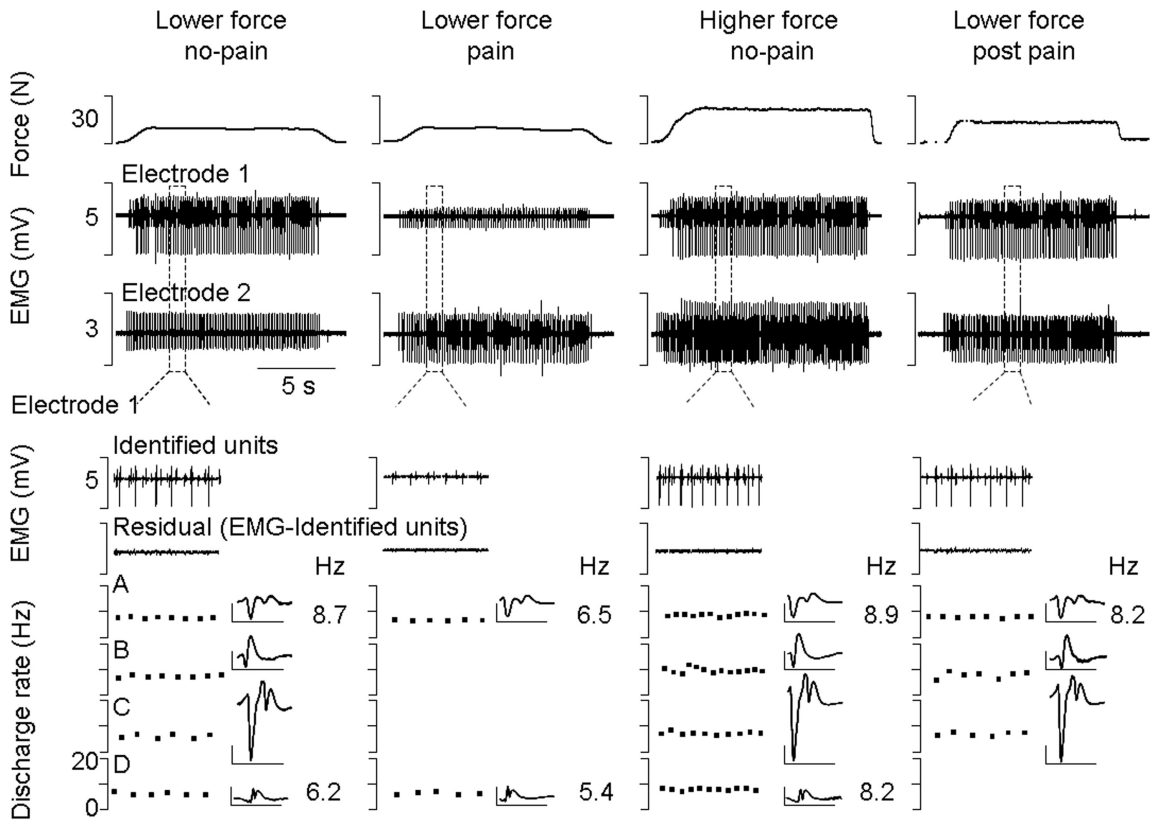

Electrode 2
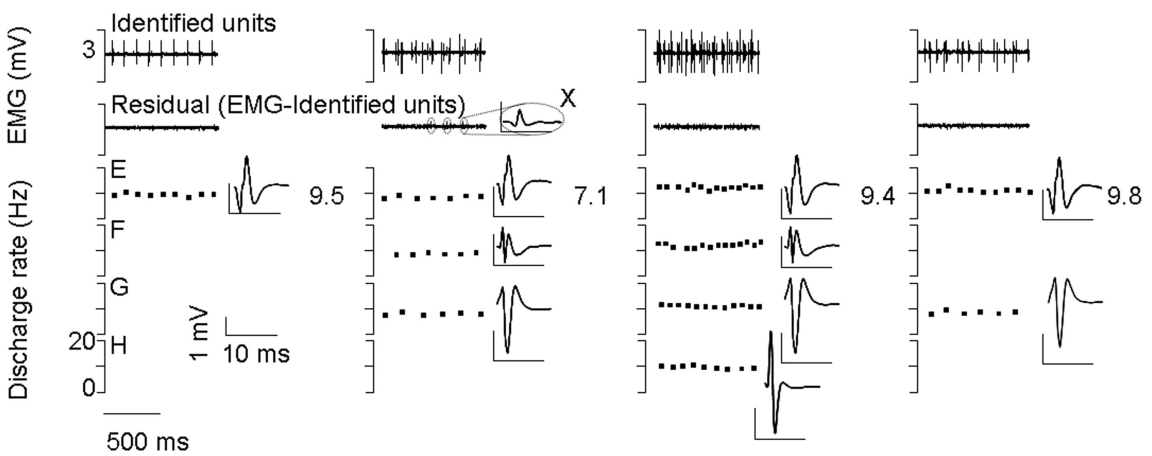

Figure 4. Simultaneous recordings of force and motor unit activity from two fine-wire electrodes inserted into the quadriceps of one subject. The top trace shows the force level during the three experimental conditions and the second and third traces show the muscle activity (EMG) recorded throughout the $\sim 15$ s constant force contractions. These traces show the consistency in force held and motor unit firing within, and variability of motor unit firing between conditions. An example of a low-force contraction after the pain was completely resolved (lower force post pain) is also shown (far right). Below the two EMG traces, $1 \mathrm{~s}$ of data has been shown in greater detail from both electrodes in each condition. The discharge rate and spike-trigger averaged electrical profile of each discriminated motor unit are shown. Data show the reduction in discharge rate during pain of motor units identified in the no-pain and pain low-force conditions $(A, D$, and E). The figure also shows a population of units that were recruited during the no-pain condition that were not recruited during pain ( $B$ and $C$ ). Force is maintained during pain by the recruitment of new units ( $F$ and $G$ ) that, in this case, are the next ones expected given orderly recruitment. Identification of units $B$ and $C$ before and after the pain condition confirms that shape had not changed with condition and therefore that these two units were not missed for that reason. The residual EMG may contain some very small spikes $(X)$ that are not classified or used for analysis purposes, but are not the units of interest.

and units D-F recruited during pain in quadriceps). In 38\% of these cases, at least one unit also remained firing in both conditions, which supports the stability of these wires between contractions [e.g., Figs. 5 (units A, C, and E), 6 (units B and C)].

Gross muscle activity did not change $(p=0.7)$ in the FPL between conditions [normalized to maximum EMG amplitude during either condition: no-pain 78.3 (32.2) and pain 85.2 (16.1)\%]. However, variability between subjects was large. EPL muscle activity was also recorded from six of the seven subjects. The group data showed no change $(p=0.3)$ in EPL activity during pain [normalized to maximum EMG amplitude during either condition: no-pain 75.8 (29.6) and pain 94.4 (13.7)\%]. However, there was large variability between subjects during the trials, and both increased and decreased activity were recorded.

\section{Discussion}

This study provides new insight into mechanisms underlying the effects of pain on movement. The results support our first two hypotheses; discharge rate of lowthreshold motor units decreased and new units were recruited during pain in muscles with and without synergists. The altered recruitment of units during pain is likely to account for maintenance of force despite reduced motor unit discharge rate. Inconsistent with our third hypothesis, two-thirds of the newly recruited units were not those expected based on orderly motoneuron recruitment. The change in recruitment pattern during pain did not change gross EMG, which supports our fourth hypothesis.

Our findings are inconsistent with uniform inhibition of painful muscles predicted by the pain adaptation theory (Lund et al., 1991). Force produced by contracting muscle depends on the number, contractile properties, and discharge rate of motor units (Burke, 1981; Stuart and Enoka, 1983). Consistent with our findings, most previous studies (Sohn et al., 2000; Farina et al., 2005; Hodges et al., 2008) have shown decreased motor unit discharge rate during pain when force was maintained. Birch et al. (2000) showed no change in discharge rate when averaged across the whole pool, but this could be explained by recruitment of new units and the increase in discharge rates of a few units as identified here.

To maintain force, reduced motor unit discharge rate must coincide with either changes in muscle fiber contractile properties and/or additional changes in motor unit recruitment within the test muscle or its synergists or antagonists. Changes in contractile properties of slowed motor units during pain cannot account for force maintenance (Farina et al., 2008). Increased synergist muscle activity has also been excluded as discharge rate decreased in medial gastrocnemius and soleus during force-matched contractions when pain was induced in lateral gastrocnemius (Hodges et al., 2008). Further, we show reduced discharge rate in a muscle without synergists (FPL).

Consistent with the pain adaptation theory, antagonist muscle activity is increased during pain in some (Arendt-Nielsen et al., 1996; Graven-Nielsen et al., 1997; Svensson et al., 1997; Falla et al., 2007) but not all (Calancie and Bawa, 1986; Birch et al., 2000; Falla et al., 2007) studies. However, to maintain force with decreased motor unit discharge, antagonist muscle activity must decrease. Here we found no consistent change in antagonist muscle activity that could account for force maintenance. Variability in EPL and FPL supports the notion that motor unit discharge properties are not simply affected by generalized inhibition or 
excitation of the agonist or antagonist motoneuron pools during pain.

Our data provide evidence that the nervous system employs a different motor unit recruitment strategy to maintain force during pain, which includes the slowing and derecruitment of one population and recruitment of a new population of units. The robust nature of our findings is supported by the consistency of observations in two different muscle systems. In addition, this study is the first to show that the newly recruited units during pain are not necessarily those expected given orderly recruitment of the motoneuron pool.

Given the novel nature of these findings, the accuracy of motor unit discrimination requires consideration. This is because the morphology of motor unit action potentials can change due to wire movement, changes in muscle fiber membrane properties, or infusion of saline. These methodological concerns were excluded in five ways. First, after a motor unit was identified from selective finewire recordings, motor unit morphology was independently assessed using spiketriggered averages generated from the separate multiunit fine-wire recordings in the FPL. Consistent action potential morphology between trials using both methods increases the confidence in accurate discrimination.

Second, contractions were maintained for $<20 \mathrm{~s}$, which reduces the possibility of fatigue-mediated effects on membrane properties. Third, saline was induced in nonmuscle tissue in the quadriceps muscle, excluding direct effects in the muscle. Fourth, in many cases recruitment and derecruitment of units were observed with the same electrode as other units that discharged in both the prepain and pain conditions (Figs. 5, 6). If ability to discriminate motor unit action potentials was affected by the above-mentioned issues, all units within the same fine-wire recordings would be expected to be affected. Finally, Figure 4 shows that two units not recruited during pain were recruited again after pain subsided. If the units were lost due to a change in morphology between conditions, it is highly unlikely that they would return to the original state in later contractions.

An additional concern is that smaller motor units may become more difficult to discriminate in signals with more active units. Given that the number of active units changes between conditions, some smaller units could be lost in more complex signals. To minimize this concern,

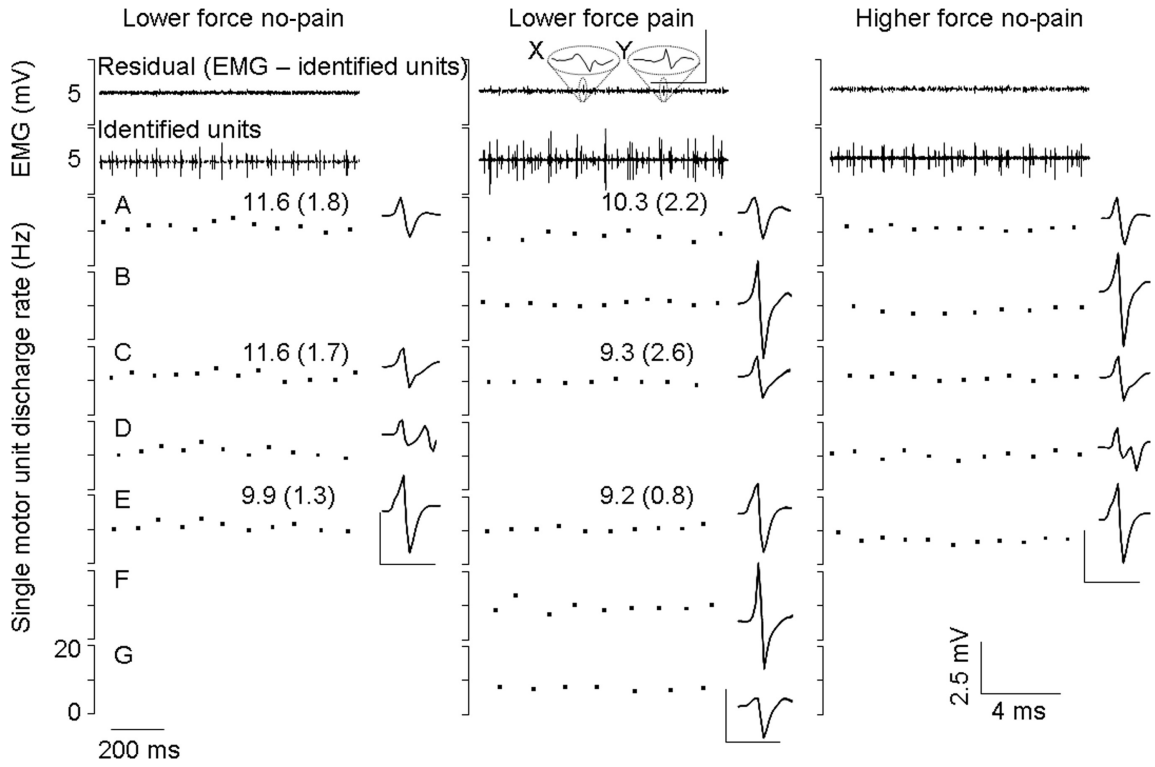

Figure 5. Recordings of motor unit activity from a fine-wire electrode inserted into the FPL of one subject. Single motor units are shown with their respective discharge rates [mean (SD)] and spike-trigger averaged electrical profile. Once these units were identified and removed from the EMG trace, there was very little residual left (top trace). An exception of two small spikes in the residual of the painful contraction is highlighted ( $\mathrm{X}$ and $\mathrm{Y}$ ). Neither spike reflects a unit that regularly discharges, and therefore they do not meet the criteria as discussed in Materials and Methods. The small residual trace clearly indicates that all reliable units were identified from this file. Three motor units were recruited during both the no-pain and pain lower-force trials $(A, C$, and $E)$. These units all decreased in discharge rate during pain. Three new units $(B, F$, and $G)$ that were not seen in the lower-force no-pain conditions were recruited during pain. Of these units, only $B$ is expected given orderly recruitment of the motoneuron pool as determined from those additional units recruited without pain at a slightly higher force level ( $B$ and $D)$. Unit $D$ is clearly not recruited during pain. The derecruitment of unit $D$, which coincides with the recruitment of $B, F$, and $G$ during pain, demonstrates a change in recruitment during pain.

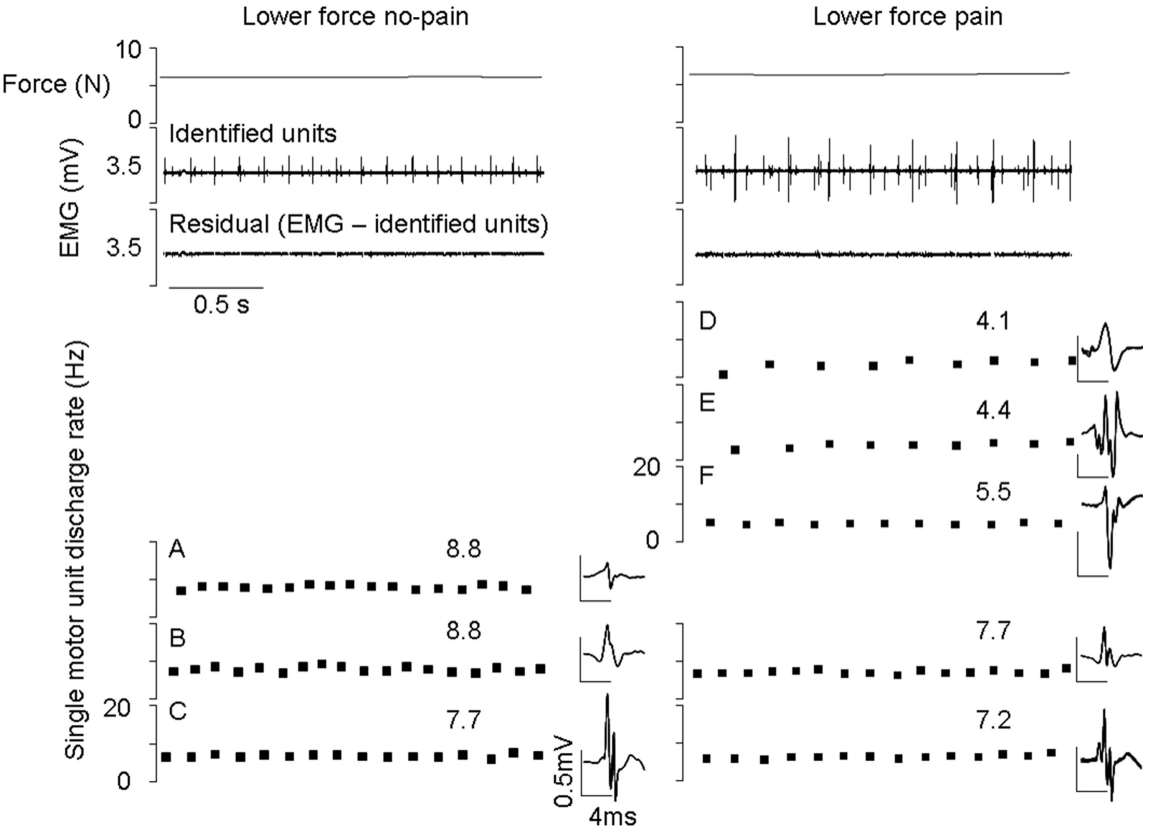

Figure 6. Recordings of motor unit activity from a fine-wire electrode inserted into the quadriceps of one subject. Single motor units are shown with their respective discharge rates and spike-trigger averaged electrical profile. The small residual EMG trace clearly indicates that all reliable units were identified from this file. Two motor units were recruited during both the no-pain and pain lower-force trials (B and C); their motor unit firing rate decreased during pain. Three new units (D-F) that were not seen in the low-force no-pain conditions were recruited during pain. Unit $A$ is not recruited during pain. The derecruitment of unit $A$, which coincides with the recruitment of D-F during pain, demonstrates a change in recruitment during pain. 
only fine-wire recordings with good signal-noise ratio in each contraction were used for analysis, and residual EMG signals were evaluated to ensure target units were not overlooked (e.g., Figs. 4-6).

To explain our observations, we propose an alternative to previous pain theories. Our new hypothesis is that rather than uniform inhibition or facilitation of the motoneuron pool, motor unit recruitment strategy is reorganized during pain. We suggest two explanations. First, a change in recruitment order, and second, a change in the population of units recruited to those with a slightly different force direction.

Motor unit recruitment order is relatively fixed, in most contexts, based on motoneuron size and common drive to the motoneuron pool (Henneman, 1957; Heckman and Binder, 1990; Burke, 1991; Mendell, 2005). This theory predicts recruitment of units from smallest to largest (Desmedt and Godaux, 1977; Veale, 1978). Departures in recruitment order have been identified due to nonphysiological stimuli (Garnett and Stephens, 1981; Semmler and Türker, 1994), changes in rate of force change, and in some voluntary tasks (Thomas et al., 1978; ter Haar Romeny et al., 1982; Riek and Bawa, 1992; Butler et al., 1999).

For true changes in recruitment order to be considered, this change must occur within the same muscle compartment, rather than different recording zones within a muscle. Our data were collected from numerous subjects and fine wires reflecting 20 fine-wire recording zones in the quadriceps and 7 fine-wire recording zones in the FPL. Both decreased and new recruitment were observed within the same fine-wire recording in $25 \%$ and $40 \%$ of quadriceps and FPL recordings, respectively (e.g., Figs. 5, $6)$. In some cases the newly recruited units were those recruited next at higher force levels; however, this was not always the case.

Changes in motor unit recruitment during pain may be explained by altered distribution of input to the motoneuron pool. If the pain adaptation theory is in part correct, and pain results in inhibition of the agonist motoneuron pool, then this inhibition will compete with the excitatory drive required to maintain force. Inhibitory input to the motoneuron pool may evoke larger IPSPs in smaller motoneurons (Lüscher et al., 1979), effectively overcoming the excitation to these motoneurons first (De Luca, 1985). This would result in slowing and/or derecruitment of lowthreshold motor units, higher levels of central drive, and subsequent recruitment of new, higher-threshold units to maintain force at prepain levels. In addition, Renshaw cell-mediated recurrent inhibition could slow or inhibit discharge of smaller lowthreshold motor units upon recruitment of larger units (Ross et al., 1975; Friedman et al., 1981; De Luca, 1985). Thus, inhibition due to pain and increased central drive and/or Renshawmediated inhibition of smaller low-threshold units could change both recruitment order and population of motor units recruited during force-matched painful contractions.

An alternative mechanism is that units with a different force direction are recruited during pain. This could occur alone or in conjunction with changes in recruitment order. Motor units within the same motoneuron pool can produce force in variable directions (Burke, 1991; Suresh et al., 2008), and may be associated with contractions of different type or orientation (Thomas et al., 1978; ter Haar Romeny et al., 1982; Riek and Bawa, 1992; Yang et al., 1998). It is possible that the population of units recruited during pain is modified to produce force with different mechanical properties, potentially to limit aggravation of painful tissue. This hypothesis is supported by changes in activity between finewire recording zones in the quadriceps muscle during pain. In this muscle $\sim 65 \%$ of new units recruited during pain were ob- served in different recording zones to those units newly recruited during the higher-force contractions. A change in recruitment order due to changes in the direction (or rate of change) of force may also explain the failure of 4 of the 19 units identified in the FPL at low force to discharge at the higher contraction level. Given the more complex signals in higher-force contractions, this could also be due to discrimination inaccuracy. However, as mentioned above, we took several steps to prevent this.

The combination of slowing or derecruitment of some units and recruitment of others resulted in no net change in gross EMG amplitude. Thus, interpretation of surface EMG recordings can be problematic for evaluation of changes in motor control strategies during pain because gross EMG depends on the balance between decreased and increased firing, both of which occur in a muscle during pain.

The novel findings of this study suggest that the nervous system employs a different motor unit recruitment strategy to achieve the same force output during pain. The decrease in discharge rate of low-threshold units is offset by recruitment of new units to maintain force. However, units that are newly recruited during pain were usually different from those recruited in a nonpainful larger contraction. The change in motor unit recruitment may be due to uneven distribution of synaptic input across the motoneuron pool during pain.

\section{References}

Arendt-Nielsen L, Graven-Nielsen T, Svarrer H, Svensson P (1996) The influence of low back pain on muscle activity and coordination during gait: a clinical and experimental study. Pain 64:231-240.

Bennell K, Hodges P, Mellor R, Bexander C, Souvlis T (2004) The nature of anterior knee pain following injection of hypertonic saline into the infrapatellar fat pad. J Orthop Res 22:116-121.

Birch L, Christensen H, Arendt-Nielsen L, Graven-Nielsen T, Søgaard K (2000) The influence of experimental muscle pain on motor unit activity during low-level contraction. Eur J Appl Physiol 83:200-206.

Burke RE (1981) Motor units: anatomy, physiology and functional organisation. In: Handbook of physiology, Sec I. The nervous system, Vol II. Motor system, Ed 1 (Brooks VB, ed), pp 345-422. Bethesda, MD: American Physiological Society.

Burke RE (1991) Selective recruitment of motor units. In: Motor control: concepts and issues (Humphrey DR, Freund HJ, eds), pp 5-21. New York: Wiley.

Butler JE, McKenzie DK, Gandevia SC (1999) Discharge properties and recruitment of human diaphragmatic motor units during voluntary inspiratory tasks. J Physiol 518:907-920.

Calancie B, Bawa P (1986) Limitations of the spike-triggered averaging technique. Muscle Nerve 9:78-83.

De Luca CJ (1985) Control properties of motor units. J Exp Biol 115:125-136.

Desmedt JE, Godaux E (1977) Ballistic contractions in man: characteristic recruitment pattern of single motor units of the tibialis anterior muscle. J Physiol 264:673-693.

Falla D, Farina D, Dahl MK, Graven-Nielsen T (2007) Muscle pain induces task-dependent changes in cervical agonist/antagonist activity. J Appl Physiol 102:601-609.

Farina D, Arendt-Nielsen L, Graven-Nielsen T (2005) Experimental muscle pain reduces initial motor unit discharge rates during sustained submaximal contractions. J Appl Physiol 98:999-1005.

Farina D, Arendt-Nielsen L, Roatta S, Graven-Nielsen T (2008) The paininduced decrease in low-threshold motor unit discharge rate is not associated with the amount of increase in spike-triggered average torque. Clin Neurophysiol 119:43-51.

Friedman WA, Sypert GW, Munson JB, Fleshman JW (1981) Recurrent inhibition in type-identified motoneurons. J Neurophysiol 46:1349-1359.

Garnett R, Stephens JA (1981) Changes in the recruitment threshold of motor units produced by cutaneous stimulation in man. J Physiol 311:463-473.

Graven-Nielsen T, Svensson P, Arendt-Nielsen L (1997) Effects of experi- 
mental muscle pain on muscle activity and co-ordination during static and dynamic motor function. Electroencephalogr Clin Neurophysiol 105:156-164.

Heckman CJ, Binder MD (1990) Neural mechanisms underlying the orderly recruitment of motoneurons. In: The segmental motor system (Binder MD, Mendell LM, eds), pp 182-204. New York: Oxford UP.

Henneman E (1957) Relation between size of neurons and their susceptibility to discharge. Science 126:1345-1347.

Hodges PW, Ervilha UF, Graven-Nielsen T (2008) Changes in motor unit firing rate in synergist muscles cannot explain the maintenance of force during constant force painful contractions. J Pain 9:1169-1174.

Le Pera D, Graven-Nielsen T, Valeriani M, Oliviero A, Di Lazzaro V, Tonali PA, Arendt-Nielsen L (2001) Inhibition of motor system excitability at cortical and spinal level by tonic muscle pain. Clin Neurophysiol 112:1633-1641.

Lund JP, Donga R, Widmer CG, Stohler CS (1991) The pain-adaptation model: a discussion of the relationship between chronic musculoskeletal pain and motor activity. Can J Physiol Pharmacol 69:683-694.

Lüscher HR, Ruenzel P, Henneman E (1979) How the size of motoneurones determines their susceptibility to discharge. Nature 282:859-861.

Mendell LM (2005) The size principle: a rule describing the recruitment of motoneurons. J Neurophysiol 93:3024-3026.

Riek S, Bawa P (1992) Recruitment of motor units in human forearm extensors. J Neurophysiol 68:100-108.

Ross H, Cleveland S, Haase J (1975) Contribution of single motoneurons to Renshaw cell activity. Neurosci Lett 1:105-108.

Semmler JG, Türker KS (1994) Compound group I excitatory input is differentially distributed to motoneurons of the human tibialis anterior. Neurosci Lett 178:206-210.
Sessle BJ (1999) Neural mechanisms and pathways in craniofacial pain. Can J Neurol Sci 26 [Suppl 3]:S7-S11.

Sohn MK, Graven-Nielsen T, Arendt-Nielsen L, Svensson P (2000) Inhibition of motor unit firing during experimental muscle pain in humans. Muscle Nerve 23:1219-1226.

Stuart DG, Enoka RM (1983) Motoneurons, motor units, and the size principle. In: The clinical neurosciences (Rosenberg RN, ed), pp 471-517. New York: Churchill Livingstone.

Suresh NL, Kuo AD, Heckman CJ, Rymer WZ (2008) Spatial variations in motor unit forces of the FDI. In: Australian Neuroscience Society 28th Annual Meeting, p 70. Hobart, Australia: Australian Neuroscience Society.

Svensson P, Houe L, Arendt-Nielsen L (1997) Bilateral experimental muscle pain changes electromyographic activity of human jaw-closing muscles during mastication. Exp Brain Res 116:182-185.

Svensson P, De Laat A, Graven-Nielsen T, Arendt-Nielsen L (1998) Experimental jaw-muscle pain does not change heteronymous $\mathrm{H}$-reflexes in the human temporalis muscle. Exp Brain Res 121:311-318.

ter Haar Romeny BM, Denier van der Gon JJ, Gielen CC (1982) Changes in recruitment order of motor units in the human biceps muscle. Exp Neurol 78:360-368.

Thomas JS, Schmidt EM, Hambrecht FT (1978) Facility of motor unit control during tasks defined directly in terms of unit behaviors. Exp Neurol 59:384-397.

Veale JL (1978) Renshaw cell activity in the human spinal cord. In: Studies in neurophysiology, pp 211-223. Cambridge, UK: Cambridge UP.

Wood Jones F (1941) The principles of anatomy as seen in the hand. London: Baillere, Tindall and Cox.

Yang D, Morris SF, Sigurdson L (1998) The sartorius muscle: anatomic considerations for reconstructive surgeons. Surg Radiol Anat 20:307-310. 